\title{
USER ACTIVITIES OUTLIERS DETECTION; INTEGRATION OF STATISTICAL AND COMPUTATIONAL INTELLIGENCE TECHNIQUES
}

\author{
SaWsan Mahmoud, Ahmad Lotfi, Caroline Langensiepen \\ School of Science and Technology \\ Nottingham Trent University \\ Nottingham, NG11 8NS, United Kingdom
}

\begin{abstract}
In this paper, a hybrid technique for user activities outliers detection is introduced. The hybrid technique consists of a two-stage integration of Principal Component Analysis (PCA) and Fuzzy Rule-Based Systems (FRBS). In the first stage, the Hamming distance is used to measure the differences between different activities. PCA is then applied to the distance measures to find two indices of Hotelling's $T^{2}$ and Squared Prediction Error. In the second stage of the process, the calculated indices are provided as inputs to the FRBSs to model them heuristically. The model is used to identify the outliers and classify them. The proposed system is tested in real home environments, equipped with appropriate sensory devices, to identify outliers in the activities of daily living of the user. Three case studies are reported to demonstrate the effectiveness of the proposed system. The proposed system successfully identifies the outliers in activities distinguishing between the normal and abnormal behavioural patterns.
\end{abstract}

Key words: Intelligent Environments, Smart Homes, Outlier Detection, Abnormal Behaviour, Principal Component Analysis, Fuzzy Rule-Based System, Distance Measure.

\section{INTRODUCTION}

To reduce the cost of care for elderly people and especially the elderly suffering from dementia, Ambient Assisted Living (AAL) is the ultimate solution to help the elderly people to live in their own home for as long as possible. It is vital to develop a good understanding of normal behaviour so as to distinguish any abnormalities or outliers, possible trends in Activities of Daily Living (ADL) and any behavioural changes. The anomaly detection system should be able to apply limited experience of environmental event history to a rapidly changing environment, taking into consideration the temporal relationships between the events. For instance, if the refrigerator door is not opened by the occupant during the day as he/she normally does, then this behaviour is considered as abnormal and an alarm should be sent to the carer. Also, if the occupant turns on the bathroom tap and does not turn it off before going to sleep, then the carer should be informed. If possible, a home controller should be activated to turn the tap off (Jakkula and Cook, 2011). Therefore, it is very important to find ways of identifying outliers or anomalies within sensor data representing the behavioural patterns and show how they affect the data analysis.

Outliers are those data that are distinct from other data points. Outliers or abnormal behaviour can cause problems in the performance of any system. An outlier detection technique could help in increasing the accuracy of the data analysis which will result in a better understanding of the information available from the data (Jain et al., 2006; Jakkula and Cook, 2010). For example, in a home environment, early detection of any anomalies in the behaviour of the occupant would lead to further investigation and increased understanding of the behavioural pattern of the user.

Address correspondence to Ahmad Lotfi, School of Science and Technology, Nottingham Trent University, Nottingham, NG11 8NS, United Kingdom; e-mail: ahmad.lotfi@ntu.ac.uk 
In this paper, a two-stage user activities outliers detection system is introduced. The proposed user activities outliers detection is comprised of the integration of statistical techniques namely Principal Component Analysis (PCA) and computational intelligence techniques namely Fuzzy Rule-Based System (FRBS). This integration will help to produce meaningful information based on a large set of historical sensory data representing the behavioural patterns of the occupant. In the first stage of the proposed process, the Hamming distance (Choi et al., 2010) is used to measure the distances between the activities. PCA is then applied to the distance measures to find two indices of Hotelling's $T^{2}$ and Squared Prediction Error. In the second stage of the process, the calculated indices are provided as inputs to FRBS to model them heuristically.

The proposed system is implemented in home environments equipped with appropriate sensory devices. The sensory devices produce long sequences of data as binary time series, indicating presence or absence of the occupant in different areas. The proposed system is used to identify outliers and then classify how far the outliers are from the remaining normal data. Three case studies are reported to demonstrate the effectiveness of the proposed system.

An outline of results of this work was presented earlier in (Mahmoud et al., 2012). The earlier paper has presented the concept of integration briefly. However, this paper formally defines the integration of PCA and FRBS and elaborates on the consequences of the proposed idea. This paper is organized as follows; Section 2 presents a summary of the related works on the identification of outliers in a smart home environment. In Section 3, our proposed outliers detection algorithm is introduced with a brief description of different components in the algorithm. Our data collection system and some detail of three case studies are explained in Section 4. The implementation of the proposed algorithm and some pertinent results are presented in Section 5. Finally, conclusions are drawn in Section 6.

\section{RELATED WORKS}

This section will review related works in two main categories of activity recognition and anomaly detection.

\subsection{Activity Recognition}

Human activity recognition is used to model behaviour in an intelligent environment (smart home) or based on wearable sensors (Lara and Labrador, 2013). The key research challenge is to find a method or an algorithm that can efficiently recognize and model human activities behaviour based on the low level sensory data. Most research carried out in this area deals with behaviour activity recognition using statistical techniques. For example, Naive Bayesian classifiers are used by Tapia et al. (2004) to classify and detect activities related to "tape on and forget" sensors. The authors used two types of activity recognition classifiers: multi-class naive classifier and multiple binary naive classifiers. The first classifier represents all activities that are needed to recognize while the second classifier represents an activity to be recognized. The major problem of Bayesian belief networks is the inflexibility of exact probabilistic inference (Li et al., 2008; Atallah and Yang, 2009; Park et al., 2009; Yogurtcu et al., 2006). Hidden Markov Model (HMM) is another statistical technique which is used to model human behaviour ( $\mathrm{Li}$ et al., 2008). Hidden Markov model is widely used to identify the activities of a user from sensor data. The main issues when using basic HMM are the difficulties experienced in processing large sets of sensory data (i.e. temporal data from different time scales). Also, for each individual activity, the sequence of sensor event cannot be separated using these models (Li et al., 2008; Singla et al., 2010).

As an alternative to the statistical methods, computational intelligence techniques are widely used to recognise ADL. For example, Multi-Layer Perceptron (MLP) neural networks 
are used to identify the movement data collected from a wireless sensor network (Cook and Tseng, 2006). For instance, Cook (2007) applied different algorithms to recognize the age categories of data representing walking pattern and to identify the change in volunteer's behaviour change. These algorithms are: MLP, decision tree, support vector classifier, Naive Bayes and Bayesnet. MLP gives the highest accuracy in classifying the categories, although the size of training and testing data sets is small. Zheng et al. (2008) proposed a special kind of Self-Organising Map (SOM) for clustering the ADL. The proposed self-adaptive neural network is called a Growing Self-Organising Map (GSOM). Using GSOM, significant activity patterns in the data along with unusual data and abnormal behaviour can be revealed and detected. One major drawback of this approach is that the optimal learning parameters need to be known a priori such as an initial learning rate and the initial neighbourhood size.

Fuzzy Rule-Based System (FRBS) is also used to model vague or uncertain data in sensor networks (Manjunatha et al., 2008; Medjahed et al., 2009a). For example, an ADL recognition system is proposed in (Medjahed et al., 2009b) using fuzzy logic to monitor the ADL of an occupant to offer him/her a safe, comfortable and appropriate environment. However, in that research the fuzzy logic was applied only on the simulated data. Also, in (Akhlaghinia, 2010), a fuzzy predictor model is used to build the prediction model and then the results are compared with the traditional time series prediction models such as ARMA, adaptive network-based fuzzy inference system and transductive neuro-fuzzy inference model with weighted normalization. One of the limitations of this work is that the proposed techniques were not apply to complex and noisy data over a long period of time. In (Doctor et al., 2005), a fuzzy learning and adaptation approach for agents called an Adaptive On-line Fuzzy Inference System (AOFIS) is proposed for ubiquitous computing environments. This approach consists of five phases including: monitoring the users' behaviour, capturing the actual data associated with their activities, computing the fuzzy membership functions from the input/output data, generating the fuzzy rules from the data and the agent control the learning and adaptation process. Then the intelligent agent has learnt, predicted and adapted to the needs of the user. However, the experiments were conducted on only five days in the intelligent Dormitory (iDorm) real environment and the results show that AOFIS results produce lower error predictions than both adaptive neuro fuzzy inference system and genetic programming.

\subsection{Anomaly Detection}

Detecting anomalous behaviour within an occupant's daily activities is a challenging task. Anomaly detection, also called outlier detection, has been an important research area in many application domains. The general definition of anomaly detection is the problem of finding pattern in data that do not conform to expected behaviour. The non-conforming patterns are often referred to as anomalies or outliers (Ye et al., 2010). Different statistical outliers detection techniques including mean, standard deviation and z-score (also known as standard score) are proposed. For example, the z-score technique is used in (Jain et al., 2006) to detect outliers in ADL. The results were generated and tested using a volunteer in an apartment setting. As such data is not distributed normally, mean and standard deviation are not good measures for detecting outliers. An alternative approach is the box plot which is a graphical representation approach for examining data sets. For example, box plot is used in (Das et al., 2010) to identify outliers which are found unusually far from the main body of the data. The advantages of the box plot are that it can display differences between populations without making assumptions about the underlying statistical distribution, and the distance between the parts of the box indicates the degree of spread and skewness in the data set. Hidden Markov Model (HMM) is also used in (Khan et al., 2012) to discover any abnormal 
behavioural patterns based on sensor networks in both indoor and outdoor settings. Three HMM approaches are used; the first approach is used on the normal activities. The second approach puts all normal activities into one class and the abnormal activities are discovered based on a threshold. In the third approach, a HMM is trained on the normal activities while the abnormal activities are approximated by using the covariances of the data points of the normal activities. In that work, all the data points from the normal events are used for training while the abnormal events are used for testing.

Artificial Neural Networks (ANN) are used to distinguish between normal and abnormal human behaviour patterns from low-level sensors. For example, Illingworth et al. (2006) demonstrated that abnormalities can be related to the temporal order in which the behavioural patterns activities happened. In their study, a temporal ANN based embedded agent is proposed. Experiments are conducted on real-time data collected from low level sensors and actuators. Also, abnormalities in a user's behavioural patterns are detected when new activities appear within these patterns.

In (Breunig et al., 2000), a Local Outlier Factor (LOF) is introduced to find outliers in multidimensional data. It is local since it allocates each object a degree to which an object is isolated from its surrounding neighbourhood. One limitation of using LOF is how to illustrate or explain why the identical local outliers are exceptional. For high dimensional data, this is essential because a local outlier may be outlying only in some, but not in all dimensions.

Anomalous behaviours or outliers are also detected using distance and similarity functions. A considerable amount of literature has been published on similarity or distance measures including Rawat et al. (2006); Huang (2008) and Yan et al. (2010). These studies investigated the usage of these measures on a wide range of applications. However, there is limited reported research so far applying these measures in an intelligent environment application, and specifically human behavioural pattern identification. Ye et al. (2010) applied the $T^{2}$ test of Mahalanobis distance to identify the outliers and anomalous events in temporal data in an intelligent environment. The data set is generated using a kernel smoothing method to give the results as a one week template. These results can be used to present the daily activities in intelligent environments. Rashidi et al. (2011) used Levenshtein "edit" distance to find the similarity between two patterns. The edit distance between two sequences is the number of edits (insertions, deletions, and substitutions) that are required to convert a sequence into another sequence. In that work, a new mining technique, called discontinuous varied-order mining method is also proposed to find the frequent patterns that might be irregular and inconsistent in the ordering. To group the patterns into activity definitions, a clustering technique is used in which the cluster centroids corresponds the activities that are identified and recognized. In a study by Jakkula and Cook (2010), an outlier detection system is proposed. They used K-Nearest Neighbour (KNN) algorithm where Discrete Time Warping (DTW) is used for distance measures. It has been proved that KNN performs well to differentiate between normal and abnormal data in intelligent environments. They argue that DTW is better than the standard distance measures such as Euclidean, Manhattan, and Chebyshev measures.

In contrast with the reported research in this area, although PCA and FRBS have been frequently used separately, our combination of PCA and FRBS approach would be useful to reduce the long series of binary data, identify the outliers and also classify the outliers. The approach not only classifies outliers or unusual activities but measures the severity of the outliers. This is important for a medical assistant or caregiver to estimate the progress or deterioration in these activities and to provide an early warning when an abnormal behaviour is expected to happen in near future. In addition, in comparison to works such as Cook (2007) and Medjahed et al. (2009b), which have used relatively small or simulated data generated from sensor networks, our work can validate and test the proposed approach on data collected from three real environments. 


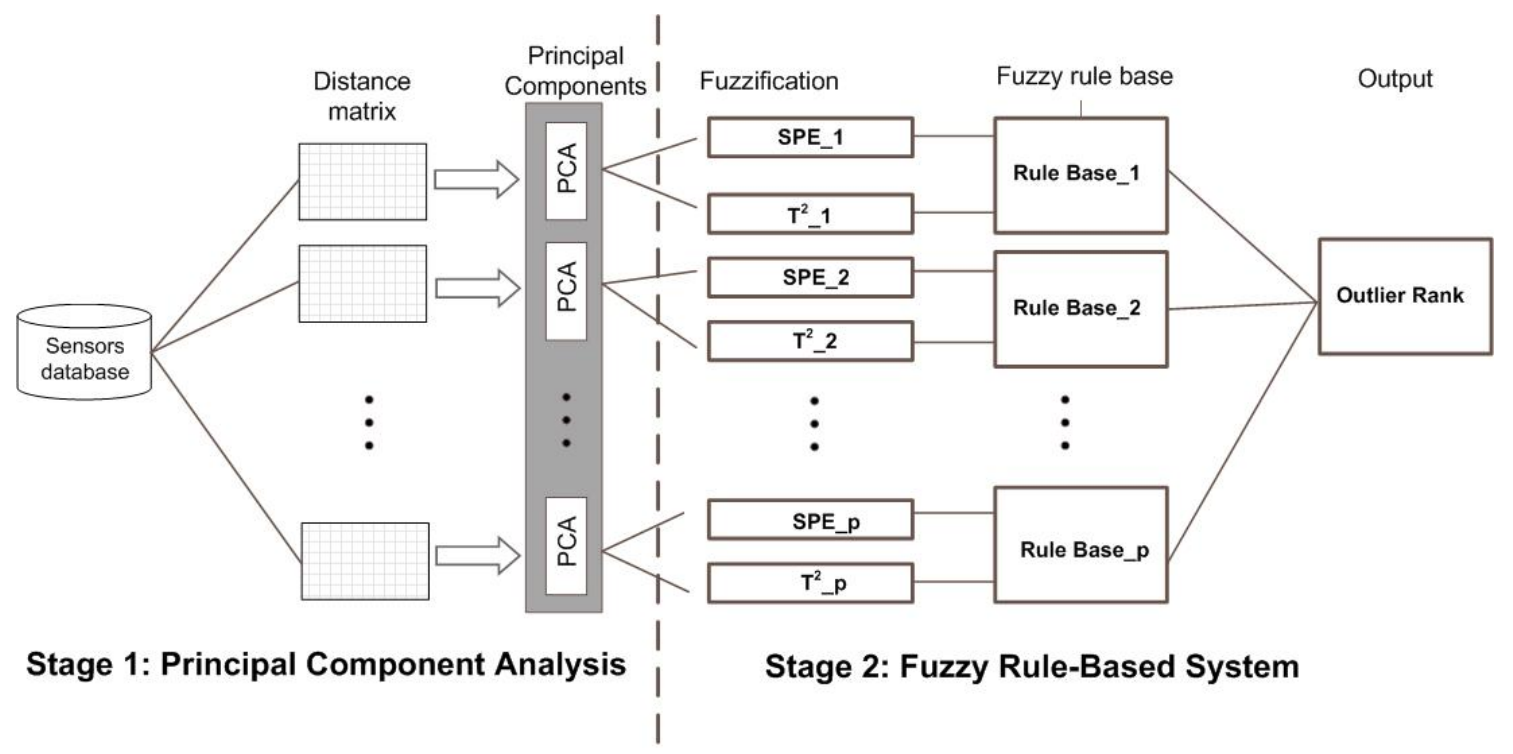

FIGURE 1: Architecture of the proposed outliers detection system.

\section{USER ACTIVITIES OUTLIERS DETECTION}

To be able to identify user activities and eventually detect any anomalies in the behaviour, the assumption is that the user is living in an environment equipped with appropriate sensory devices. The information gathered from occupancy sensors (including PIR and door entry sensors) and electrical appliances would provide basic behavioural information. Therefore by monitoring the sensor data, important information regarding any irregular behaviour will be identified. In some other applications, these kinds of patterns are also called outliers, discordant observations, exceptions, surprises or peculiarities. Amongst all mentioned terminology, anomalies and outliers are the most frequently used terms within the context of human behaviour detection (Chandola et al., 2009).

The architecture of the proposed outliers detection system is shown in Figure 1. Components of the proposed system to identify the outliers are listed below:

- Collect the sensor data from the environment,

- Calculate the distance matrices for the collected data using a distance measure,

- Conduct the PCA for the distance matrices. The output from the PCA are Hotelling's $T^{2}$ and Square Prediction Error (SPE) indices,

- Calculate confidence limits for both Hotelling's $T^{2}$ and SPE indices,

- Compute the degree of memberships for both Hotelling's $T^{2}$ and SPE indices as the inputs of Fuzzy Rule-Based Systems. Confidence limits identified in the previous step define the universe of discourse for the inputs,

- Formulate the rules in the FRBSs and check whether inputs exceed the confidence limits,

- Generate the output values to provide a rank for data point to indicate the membership degree of the outliers.

The proposed outliers detection system is based on a two-stage process where the first stage includes a dimensionality reduction of data in which PCA is used. Unlike most machine learning and data mining techniques, PCA efficiently reduces the high multi-dimensional data into a new lower dimensional representation. PCA is able to extract useful features from data sets and help in producing a better visualization of the data. It does this by transforming the data in a way that shows the maximum variability within the data. The 
second stage includes an outlier or abnormality identification using FRBSs. More details about the components described above are presented in the following sections.

\subsection{Distance Measures}

Distance or dissimilarity measures are used to find the degree of differences between two sets of data. Generally, finding dissimilarities between two vectors mean that both vectors have changes in their patterns. The values that have common patterns are considered to be closer to one another than those with different patterns (Huang, 2008).

For example, consider the occupancy sensors data collected from a real environment. It is usually presented as a long sequence of binary data. If we want to compare or measure the distance between two sequences, there are many distance measures suggested in the literature (Huang, 2008; Cha et al., 2005; Erlich et al., 2002; Rawat et al., 2006; Yan et al., 2010).

The most commonly used distance measure between two binary feature vectors is Hamming distance (Choi et al., 2010). Hamming distance can be defined as the number of mismatching bits between two binary vectors of the same length. Formally, let $A$ and $B$ be two binary vectors i.e. have the $i^{t h}$ feature value either 0 or $1 . A$ and $B$ are representing two sets of binary data collected for two separate periods of time for the same sensor. The Hamming distance $D$ between $A$ and $B$ is computed as follows:

$$
D(A, B)=S_{01}+S_{10}
$$

where $S_{01}$ is the number of occurrences of matches with 0 in the first pattern and 1 in the second pattern at the corresponding positions. $S_{10}$ is the number of occurrences of matches with 1 in the first pattern and 0 in the second pattern at the corresponding positions. Distance measures can be used to find regular behaviour patterns and eventually any deviation from one day to another. If the result of the distance $D(A, B)$ is very small, the two binary vectors are similar. Otherwise, the vectors are dissimilar.

To illustrate the process, Figure 2 shows door opening/closing activities for two different days (this is the back door in case study discussed in Section 4). In Figure 2-a, on that day the back door is only opened once (bit state 1) and it is closed most of the time (bit state 0). In Figure 2-b, on that day the back door is also closed most of the time although it is opened more than one time. In this case, the Hamming distance is a good choice since it takes into consideration the mismatching bits only and ignores the matches of the negative state (i.e. 0 bits).

\subsection{Dimensionality Reduction}

Behavioural information is collected from low level (binary) sensors, which consist of long series of multi-dimensional data. The dataset is usually sparse and contain many repeated values. Identifying outliers and abnormality in a high dimensional matrix is a complex process compared with a low dimensional matrix. The outliers in multi-dimensional data do not appear by using each individual dimension i.e. they are not identified using a univariate approach (in this approach outliers are detected when the standardized value of the data point is large). Therefore, it is better to handle the outliers using a multivariate approach where outliers are detected considering all features of the multi-dimensional data. An approach which efficiently reduces the high dimensionality of the data is required for better visualization and ultimately identification of any outliers or abnormality.

There are many methods that can be employed for dimensionality reduction to analyse high dimensional data (Han and Kamber, 2006; Cunningham, 2007). These methods can be generally classified into supervised and unsupervised. The classification is based on whether the supervision information is used or not. Linear Discriminate Analysis (LDA) is the most 


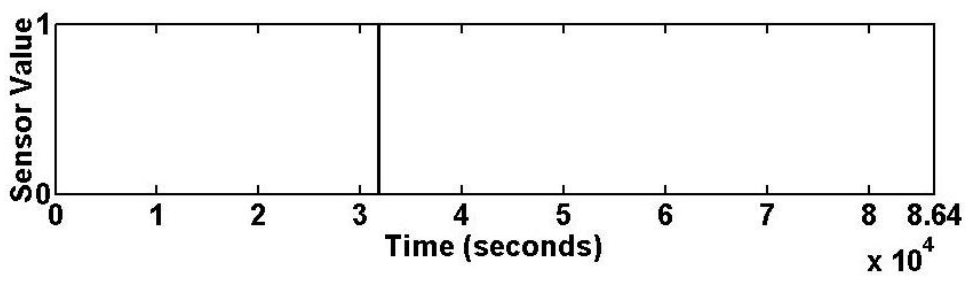

(a) Day 1

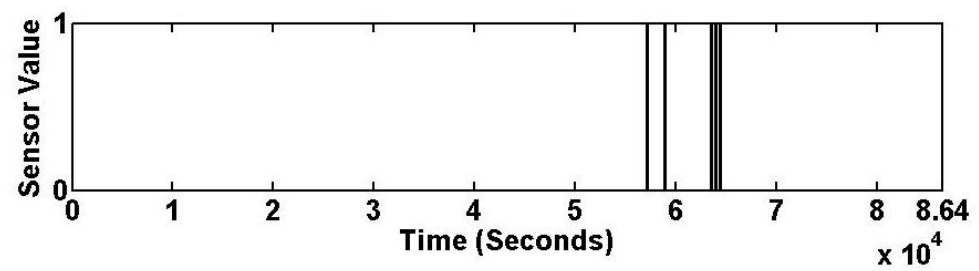

(b) Day 2

FIGURE 2: A sample of two days of the back door entry point data collected from the environment in case study I. (a) Day1, (b) Day2.

widely used supervised approach to discriminate and reduce the dimensionality of data. LDA accomplishes maximum class discrimination through minimizing the "within class" distance and maximizing the "between class" distance at the same time (Ye and Ji, 2009). On the other hand, unsupervised reduction methods use class labels to reduce a high dimensional data where no supervision information is used. PCA is one of the most commonly used unsupervised dimensionality reduction methods (Mller et al., 2006; Chen and Zhang, 2011).

Dimensionality reduction is performed using linear or non-linear techniques depending on the type of data. With linear techniques, the data is assumed to lie on or near a linear subspace of the high-dimensional space. PCA is a linear technique which maximizes data global variance by orthogonal projection. Linear techniques cannot process complex non-linear data efficiently (Liu et al., 2005). Thus, numerous non-linear techniques have been introduced to solve the difficulties associated with linear techniques. The non-linear techniques can efficiently deal with complex non-linear high-dimensional data (van der Maaten et al., 2009). Global non-linear technique is a type of dimensionality reduction that is used to preserve global properties of the data. Multi-Dimensional Scaling (MDS), Isomap and Kernal PCA are examples of such non-linear techniques.

MDS are non-linear techniques used to map the high-dimensional data into a lowdimensional data by keeping the pairwise distances between the data points. It is generally used for data visualization. One of the disadvantages of MDS, is that it is based on Euclidean distances and does not consider the distribution of the neighbouring data points (van der Maaten et al., 2009). Isomap is a non-linear technique used to overcome the problems of MDS by preserving pairwise geodesic or (curvilinear) distances between data points. Geodesic distance is the distance between two data points. One of the major problems with Isomap is its topological instability. This problem is resolved by removing data points which have many flaws in the shortest path algorithm. In addition to MDS and Isomap, Kernel PCA is considered an alternative way of traditional linear PCA and metric MDS. It is expressed using a kernel function. In kernel PCA, the principal eigenvectors of the kernel matrix are computed instead of the covariance matrix. In this technique, the PCA is applied in kernel space which gives kernel PCA the ability to be applied on non-linear data. This technique has 
an important limitation that the size of kernel matrix is relative to the square of the number of instances in the data (van der Maaten et al., 2009; Engel et al., 2012).

In this paper, PCA is used on the high multi-dimensional distance matrix. Comparing PCA with other data reduction techniques, PCA is capable of processing sparse data efficiently and is computationally inexpensive (Mller et al., 2006). Also, the mathematical foundation of PCA is quite uncomplicated compared with LDA and DWT approaches. PCA is a more appropriate approach than LDA when the data is unlabelled (Cunningham, 2007). PCA is a linear transformation approach and the interpretation of its Principal Components (PCs) can be difficult (Cunningham, 2007). To overcome this difficulty, FRBS is used in the proposed system to summarize data and help in classifying outliers and their severity.

Formally, let us consider $X$ as an input matrix which consists of $N$ observations and $M$ variables. Standardization such as z-score is normally used when variables are measured in different units. PCA takes the input matrix and transforms it into eigenvectors and associated eigenvalues. The first PC contains the data with the highest variance while the second PC contain the data with the next highest variance and so on for other components. The format of the eigenvalues and eigenvectors are as follows:

$$
\begin{gathered}
\Lambda=\left[\begin{array}{cccc}
\lambda_{1} & 0 & \ldots & 0 \\
0 & \lambda_{2} & \ldots & 0 \\
\vdots & \vdots & \ddots & \vdots \\
0 & 0 & \ldots & \lambda_{M}
\end{array}\right] \\
V=\left[e_{1}, e_{2}, \ldots, e_{k}\right]
\end{gathered}
$$

where $\lambda_{1}, \lambda_{2}, \ldots, \lambda_{k}$ are eigenvectors, $e_{1}, e_{2}, \ldots, e_{k}$ are eigenvalues and $k$ is the number of selected PCs. Changes within the data are detected using two statistical index measures named Hotelling's $T^{2}$ and Square Prediction Error (SPE). When PCs are identified, the $T^{2}$ and SPE indices measures are computed. These two statistic measures are briefly described below.

3.2.1. Hotelling's T-Squared Statistic. Hotelling's T-Squared $\left(T^{2}\right)$ measures the squared norm of the current sample from the centre of the normal data points region (Liu et al., 2005). In other words, the $T^{2}$ index measures the variations in the PCs and it is calculated using the following expression:

$$
T^{2}=X^{T} V \Lambda^{-1} V^{T} X
$$

or

$$
T^{2}=\sum_{i=1}^{k} \frac{t_{i}^{2}}{\lambda_{i}^{2}}
$$

where $t_{i}$ is the $i^{t h}$ element in the vector $t=V^{T} X$. The limit of $T^{2}$ index with a confidence level $\alpha$ is:

$$
T_{\text {lim }}^{2}=\frac{k(N-1)}{N-k} F(k, N-k, \alpha)
$$

where the $F(k, N-k, \alpha)$ corresponds to the probability point on the F-distribution with $(k, N-k)$ degrees of freedom and confidence level $\alpha$. 
3.2.2. Square Prediction Error Statistic. The Square Prediction Error (SPE) index measures the projection of the data points on the residual subspace (Liu et al., 2005). It is calculated using the following expression:

$$
r=X^{T}-X=X^{T}-V_{k} V_{k}^{T} X
$$

and

$$
S P E=r^{T} r
$$

The residual matrix $r$ captures the variations in the observation space spanned by the PCs associated with the $M$ smallest singular values. The sub spaces spanned by $X$ and $X^{T}$ are called the score space and residual space respectively (Bouhouche et al., 2007). The limit for SPE index which denotes the upper control limit for SPE index with a confidence level $\alpha=95 \%$ is (Liu et al., 2005):

$$
S P E_{\text {lim }}=\theta_{1}\left[\frac{C_{a} h_{0} \sqrt{2 \theta_{2}}}{\theta_{1}}+1+\frac{\theta_{2} h_{0}\left(h_{0}-1\right)}{\theta_{1}^{2}}\right]^{\frac{1}{h_{0}}}
$$

where $\theta_{i}=\sum_{j=k+1}^{m} \lambda_{j}^{i}$ and $h_{0}=1-\frac{2 \theta_{1} \theta_{3}}{3 \theta_{2}^{2}}$.

The above two indices are checked as to whether they exceed their control limits. If both $T^{2}$ and $S P E$ exceed their upper limit, the process is considered as abnormal. Usually, $T^{2}$ is more sensitive to the changes or variation in a process over time that leads to move the process far from normal. $T^{2}$ is a measure of the deviation in the model subspace and $S P E$ is a measure of the deviation in the residual space. $S P E$ is applied to identify when the current operation deviates from the normal in terms of parameters that are not dominant (Kumar et al., 2008).

\subsection{Fuzzy Rule-Based System}

Fuzzy Rule-Based System can efficiently model vague or uncertain sensory data and can deal with complex data collected from different sensors (Martin et al., 2006). It can report the user's activities in terms of linguistic variables instead of the raw or pre-processed sensor data. In addition, it can give justifications for that report to be more understandable to the end user of the system.

In the second stage of the proposed outlier detection process, a group of FRBSs is used to classify and summarize users activities based on the information gathered from the installed sensors in the environment. To develop the FRBS, initially a fuzzification process is performed. This is followed by fuzzy rule generation and an inference system. In the fuzzification stage, the sensor data set is converted from its crisp values into fuzzy values by assigning the membership degrees of each value in the input and output data set (Medjahed et al., 2009b). Features extracted from the PCs are used to determine the degree of membership of each sensor data set. These features are represented by the two error indices Hotelling's $T^{2}$ and $S P E$. These indices form the input variables to FRBSs where the universe of input variables are limited to $T_{l i m}^{2}$ and $S P E_{l i m}$ as calculated in Equation 6 and Equation 9. The confidence limits for both $S P E$ and Hotelling's $T^{2}$ are computed for each sensor data set. Hotelling's $T^{2}$ and $S P E$ are labelled on a numerical scale based on these confidence limits.

For each FRBS, fuzzy rules with the following configuration are used:

$$
R_{j}^{i}: \text { If } T_{j}^{2} \text { is } \tilde{A}_{j}^{i} \text { and } S P E_{j} \text { is } \tilde{B}_{j}^{i} \text { Then outliers rank } \text { is }_{j}^{i}
$$


where $R_{j}^{i}$ is the label of $i^{\text {th }}$ rule for the sensor $j . T_{j}^{2}$ and $S P E_{j}$ are the inputs for the sensor $j$. outliersrank $_{j}$ is the output, $\tilde{A}_{j}^{i}$ and $\tilde{B}_{j}^{i}(i=1,2, \ldots, m$ and $j=1,2, \ldots, p)$ are fuzzy labels for inputs and $\tilde{C}_{j}^{i}(i=1,2, \ldots, n)$ is the label for outputs. $p$ is the number of sensor data set, $m$ is the number of labels for input Membership Functions (MFs) and $n$ is the number of labels for output MFs.

The input variables have three fuzzy MFs $\mu: \mu($ Low $), \mu($ Medium $)$ and $\mu($ High $)$. They have different values depending on the type of sensor. The output variable of the FRBS representing outliers rank is the degree of belief towards the class. Fuzzy sets are defined on this linguistic variable in a similar way as is done in the case of linguistic variables for the $T^{2}$ and $S P E$ inputs. Five MFs are defined: $\mu$ (Extremely Outlier - EO), $\mu$ (Slightly Outlier - SO), $\mu$ (Medium - M), $\mu$ (More or Less Normal - MN) and $\mu$ (Normal - NO). These MFs are created for each sensor in the environment where the universe of output variable is $[0,1]$. Figure 3 shows a sample of the membership labels for the inputs and output for one sensor (this is the back door entry sensor in case study I as explained in Section 4).

For each sensor data, fuzzy rules are built using the values of the two statistical error indices $T^{2}$ and $S P E$. If both $T^{2}$ and $S P E$ measures exceeds their control limits then the status for the process is abnormal. Otherwise, the status of the process is considered as normal when both indices are less than their limits (Liu et al., 2005). It is also possible to reach other options if none of the above are satisfied. Therefore, to reach a decision based on the values of the indices, a FRBS is used to provide the decision. Nine fuzzy rules with three membership for each inputs and five membership for each output ( $m=3$ and $n=5$ ) for outliers rank identification are defined as shown below:

R1: IF $T^{2}$ is $\mu(H i g h)$ AND $S P E$ is $\mu(H i g h)$ THEN outliers rank is $\mu(E O)$

R2: IF $T^{2}$ is $\mu(H i g h)$ AND $S P E$ is $\mu$ (Medium) THEN outliers rank is $\mu(S O)$

R3: IF $T^{2}$ is $\mu($ High $)$ AND $S P E$ is $\mu($ Low $)$ THEN outliers rank is $\mu(S O)$

R4: IF $T^{2}$ is $\mu$ (Medium) AND $S P E$ is $\mu$ (Low) THEN outliers rank is $\mu(M N)$

R5: IF $T^{2}$ is $\mu$ (Medium) AND $S P E$ is $\mu$ (Medium) THEN outliers rank is $\mu(M)$

R6: IF $T^{2}$ is $\mu$ (Medium) AND $S P E$ is $\mu($ High) THEN outliers rank is $\mu(S O)$

R7: IF $T^{2}$ is $\mu($ Low $)$ AND $S P E$ is $\mu($ High) THEN outliers rank is $\mu(M)$

R8: IF $T^{2}$ is $\mu(L o w)$ AND $S P E$ is $\mu$ (Medium) THEN outliers rank is $\mu(M N)$

R9: IF $T^{2}$ is $\mu$ (Low) AND $S P E$ is $\mu$ (Low) THEN outliers rank is $\mu(N O)$

The final outliers rank is decided based on the rank of the outliers for each sensor. The final rank is calculated as:

$$
\text { outliers rank }=\min _{j=1}^{p}\left(\text { outliers rank } k_{j}\right)
$$

These fuzzy rules are used to classify outliers and distinguish the normal and abnormal behaviour patterns. In addition, the severity of outliers are also identified. A schematic diagram of the FRBSs used for the second stage of the proposed outlier detection system is illustrated in Figure 4.

\section{DATA COLLECTION AND CASE STUDIES}

Research and development have mainly focused on the utilisation of different low-key technological devices which are readily available. Most patients would prefer to use a nonintrusive technology to help them with their day-to-day activities. For example usage of surveillance cameras for patient monitoring is not welcomed and in most cases it is ruled 


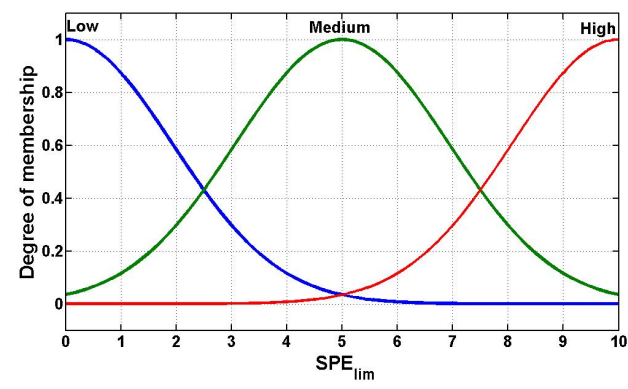

(a)

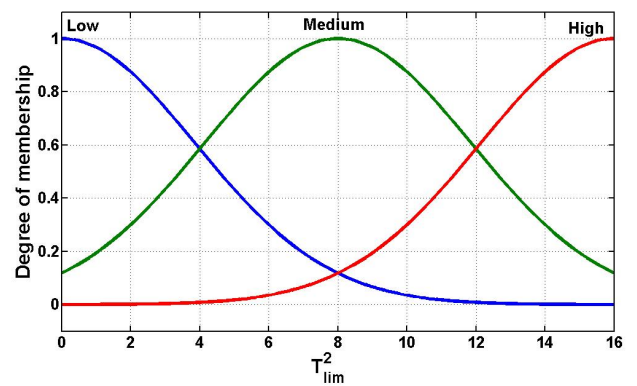

(b)

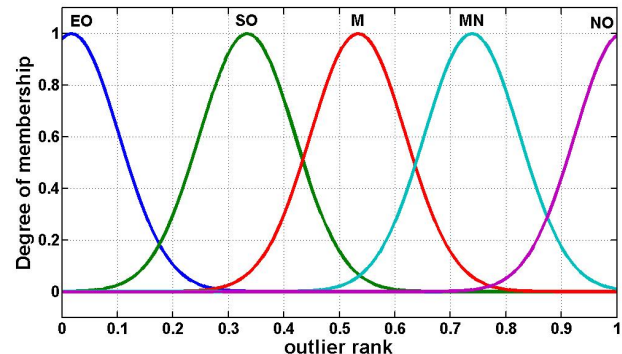

(c)

FIGURE 3: Membership labels for input and output variables for the back door sensor. (a) $S P E_{\text {lim }}$, (b) $T_{\text {lim }}^{2}$ and (c) Outlier rank

out completely (Kenner, 2008). In this study, to test and validate the proposed user activities outliers detection, real home environments equipped with appropriate sensory devices are used. Three case studies are used to elaborate on the proposed technique. In these case studies, each occupant is living alone in different real environments where their movement activities are different from one to another. More detailed description of these case studies are presented below. More information about the hardware equipment and data collection system is available from (Lotfi et al., 2012).

\subsection{Case Study I}

In this case study, the real data is collected from an environment monitored by a Just Checking Ltd. monitoring system (JustChecking, 2013). The environment is equipped with different sensors with a controller which receives data from the sensors and uploads it to a 


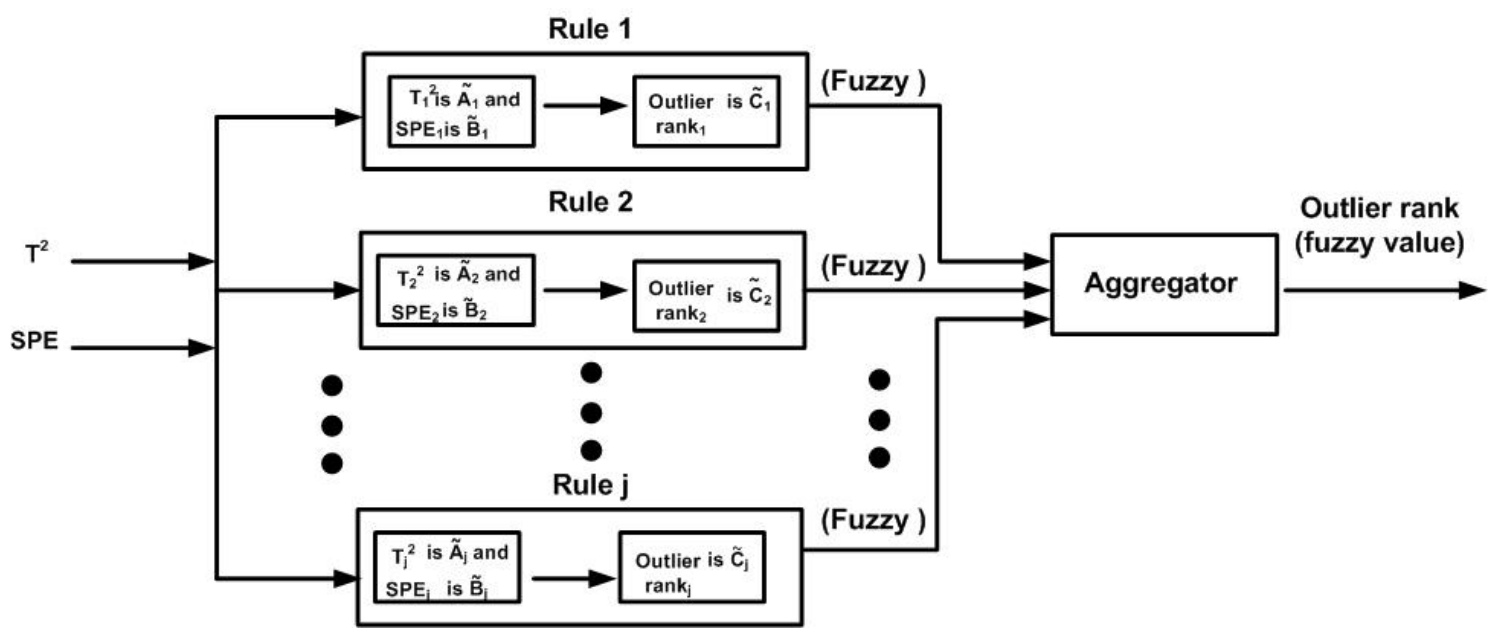

FIGURE 4: Fuzzy Inference system with two inputs and one output.

web-server via an integral mobile phone unit. These are front and back door entry sensors and lounge, kitchen, bedroom and bathroom motion sensors. The collected data from the occupancy sensors were recorded with a resolution of one second to monitor the daily behaviour of the occupant. Logged data is time stamped and includes sensor ID and a sensor name (type). The data was collected for a duration of over one year.

\subsection{Case Study II}

The data for this case study is collected from another real environment equipped with the same monitoring system in case Study I for another elderly occupant. For this environment, two door entry sensors including front door and back door as well as four motion sensors including kitchen, lounge, upstairs, and bathroom are used. The elderly person was first prescribed some medication, and her health status got worse. Consequently, she was roaming around during the early hours of the day, and her behaviour was considered as abnormal. Then her first medication was replaced by new medication and the patient's health got better. In this research, the data collected from this environment is split into two separate groups. One group represents the data when the health of the elderly got worse and the other group for the data when the health of the elderly got better.

\subsection{Case Study III}

The data for this real environment is collected from an elderly occupant living in her apartment based on a system developed by Akhlaghinia (2010). The apartment is located in a council complex in Nottingham in the United Kingdom. The elderly person uses a walker support to help her to move around her apartment where most of her ADLs are carried out. Four motions sensors covering the lounge, kitchen, bedroom and corridor are used. Additionally, two door entry sensors were used to monitor the bathroom and the main entrance doors. A sample of raw data collected from this environment is illustrated in Table 1. A data acquisition system is installed in the apartment to monitor the occupancy. A wireless receiver agent is used and put in a safe place in her flat. In addition, there is a base station to store the collected data from the wireless sensor network. Thus, the collected data from sensors are transmitted to the base station and logged in a database file using the monitoring portal on the base station. The data is collected for a couple of weeks to monitor the ADLs of the elderly person where holidays and weekends are not included. 


\section{RESULTS}

In this section, the proposed outliers detection system is validated using the case studies presented earlier. In the beginning of our analysis, the binary distance measure is computed on the raw data to construct the distance matrix. Binary distance measures are used to improve the outliers and abnormality identification. A sample of the results obtained for the Hamming distance for the kitchen occupancy sensor for case study I is shown below:

$$
D(365 \times 365)=\left[\begin{array}{ccccc}
0 & 12375 & \ldots & 10209 & 10094 \\
12375 & 0 & \ldots & 13026 & 13291 \\
9987 & 15734 & \ldots & 14686 & 14629 \\
\cdot & \cdot & \ldots & \cdot & \cdot \\
\cdot & . & \ldots & . & . \\
6664 & 11561 & \ldots & 10191 & 11620 \\
10209 & 13026 & \ldots & 0 & 12373 \\
10094 & 13291 & \ldots & 12373 & 0
\end{array}\right]
$$

where $D(i, j)$ represents the distance between day $i: 1, \ldots, 365$ and day $j: 1, \ldots, 365$. $i$ and $j$ are the indices for different days and it should be noted that $D(i, i)=0$. It is almost impossible to visualise and identify normal and abnormal behavioural patterns of the occupant from this matrix.

To select the important features for the above distance matrix, PCA is applied. The question is how many PCs should be retained for analysis? There are many ways to choose; one way is to select only those whose eigenvalue is greater than one. Another way is to apply Cumulative Percent Variance (CPV) approach. It can be calculated using the following formula:

$$
C P V(a)=\frac{\sum_{i=1}^{a} \lambda_{i}}{\operatorname{trace}(R)} 100
$$

$\mathrm{CPV}$ is a measure of the percentage of the variability $(C P V(a)>90 \%)$ captured by the first principal components. A graphical plot, called a scree plot, is also used to choose the number of PCs. In this graph, all the eigenvalues are plotted in their decreasing order and the "knee" in the graph is identified. To select the components, count the PCs between the knee and the high component. The results of the scree plots are shown in Figure 5 based on the back door entry sensor data. The first two PCs were selected. In this work, scree plot are used to choose the number of PCs to keep in the model. Only the PCs that have eigenvalues greater than 1 were selected since component with eigenvalues less than 1 have less variance than did the original value. Figure 6-a to Figure 6-d show the scatter plots for the first and

Table 1: A sample of raw data collected from the environment in case study III.

\begin{tabular}{lll}
\hline Time Stamp & Sensor:Value & Type \\
\hline 13/05/2012 16:07:12 & 11:0 & Corridor - OFF \\
13/05/2012 16:07:20 & $21: 160$ & Main door - Open \\
13/05/2012 16:07:39 & $21: 0$ & Main door - Close \\
13/05/2012 16:07:43 & $11: 160$ & Corridor - ON \\
13/05/2012 16:07:45 & $21: 160$ & Main door - Open \\
13/05/2012 16:07:45 & $11: 0$ & Corridor - OFF \\
13/05/2012 16:07:47 & $14: 255$ & Lounge - ON \\
13/05/2012 16:07:48 & $14: 0$ & Lounge - OFF \\
13/05/2012 16:07:51 & $14: 255$ & Lounge - ON \\
\hline
\end{tabular}




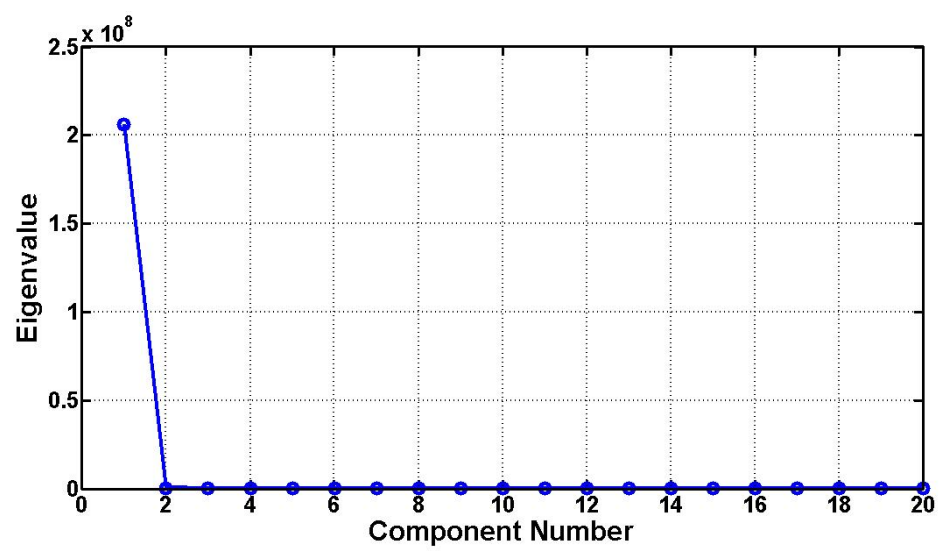

FIGURE 5: Significant number of components for PCA analysis based on data extracted from the back door entry sensor for one year.

the second PCs of the binary data collected from the environment for back door, front door, lounge motion and kitchen motion sensors respectively. In these figures, the text numbers are the days that activities are carried out. In these figures, the PCs are calculated using $\alpha=95 \%$.

PCs are used to calculate two indices namely residual $S P E$ and Hotelling's $T^{2}$ measures. The $S P E$ and Hotelling's $T^{2}$ measures for the back door sensor in case study I is shown in Figure 7. These measures are used in the second stage of the process to classify outliers within the data sets. These indices are the inputs to FRBS classifiers with one output representing the outliers rank. For case study I, the $S P E$ limits are 1.9255, 6.2451, 211.0567 and 20.9023 for back door, front door, lounge and kitchen sensor respectively. The $T^{2}$ limit is 3.8671 for all sensors since the most variations are located on the 1st and 2nd PC and $T^{2}$ index limit depends on these two PCs. Based on the granulated fuzzy labels for each sensor data, fuzzy rules are defined.

By using the fuzzy rules, the outliers and anomalies are identified along with the relative position of the outliers within the data (outliers rank). In Figure 8-a to Figure 8-d labelled PCs based on the fuzzy outliers rank are shown for the same results shown in Figure 6-a to Figure 6-d. It is obvious that the outliers are detected according to their position in the actual binary data sets. In addition, the most frequent observations (normal group of data) were selected from similar ADLs carried out during the year. However, a few observations, which are located far from the normal group of data, represent a set of individual days where ADLs are unlikely to be carried out (abnormal group). For example, three clear groups of data are recognized in Figure 8-b, these are: (1) Normal group (in fuzzy label NO and representing by triangles) represents the set of the days in the year where the back door is not often opened and closed, (2) Abnormal or outliers group (in fuzzy label EO and representing by squares), which is located to the left of the graph (i.e. day index 43), represents the data where the back door remained open for a long time, and (3) More or less normal group (in fuzzy label $\mathrm{MN}$ and representing by circles) represents the set of the days where the door is frequently opened but for a short time. It can be concluded that the proposed outliers system gives a visual grouping to the data of similar features and detects outliers and anomalies.

If there are slight outliers and changes or extreme outliers, alarm messages could be sent to the carer to help the elderly person. Figure 9-a and Figure 10-a show the scatter plots for the first and the second PCs of the binary data collected from the environment of the bedroom motion sensor and bathroom door entry sensor of case study III. The corresponding labelled 


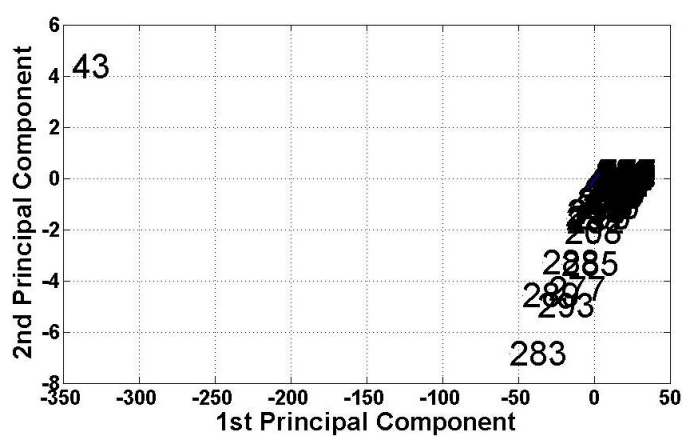

(a) Back door

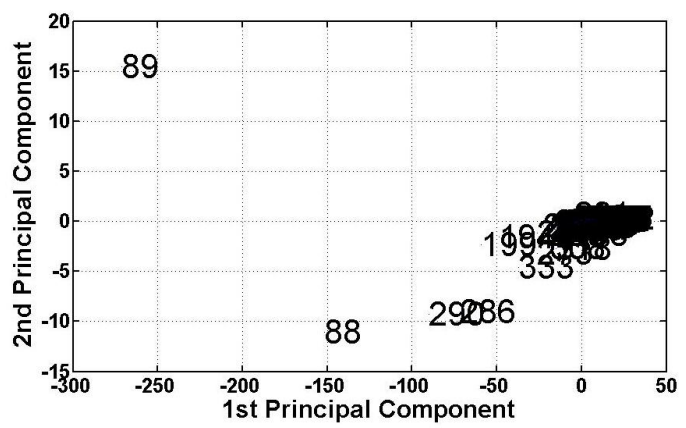

(b) Front door

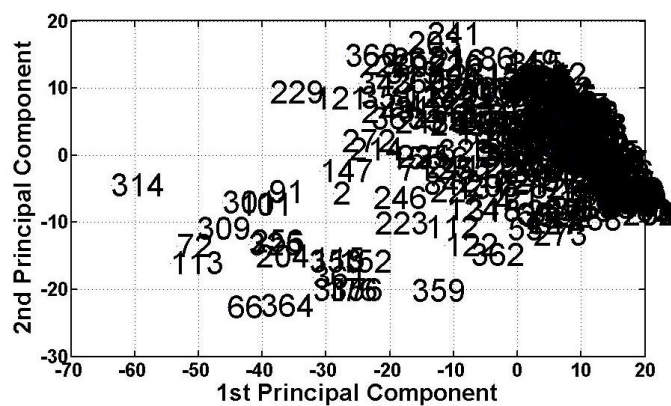

(c) Lounge

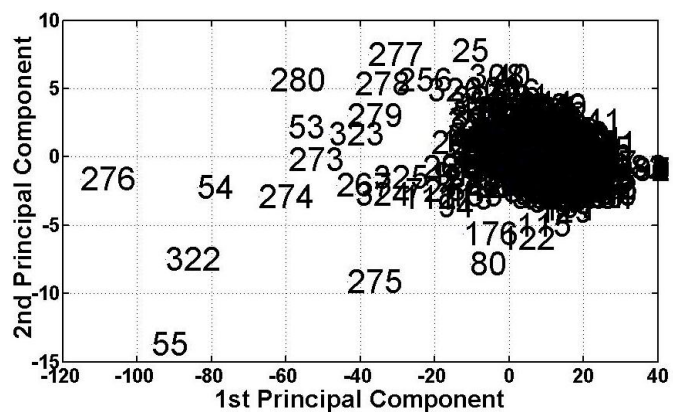

(d) Kitchen

FIGURE 6: Scattered plot for the 1st and 2nd principal components of the data used in case study I. 


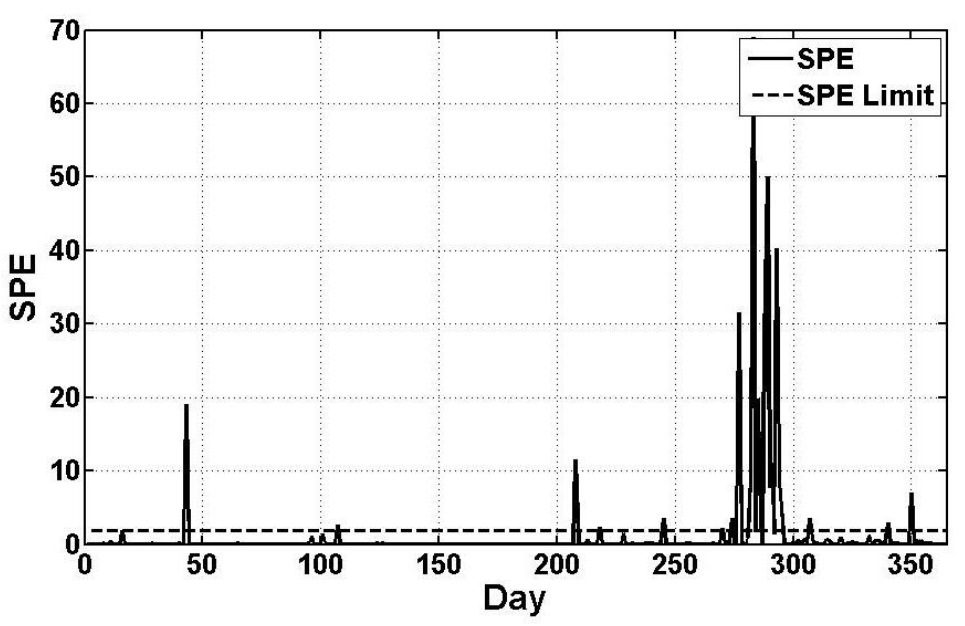

(a) $S P E$ index

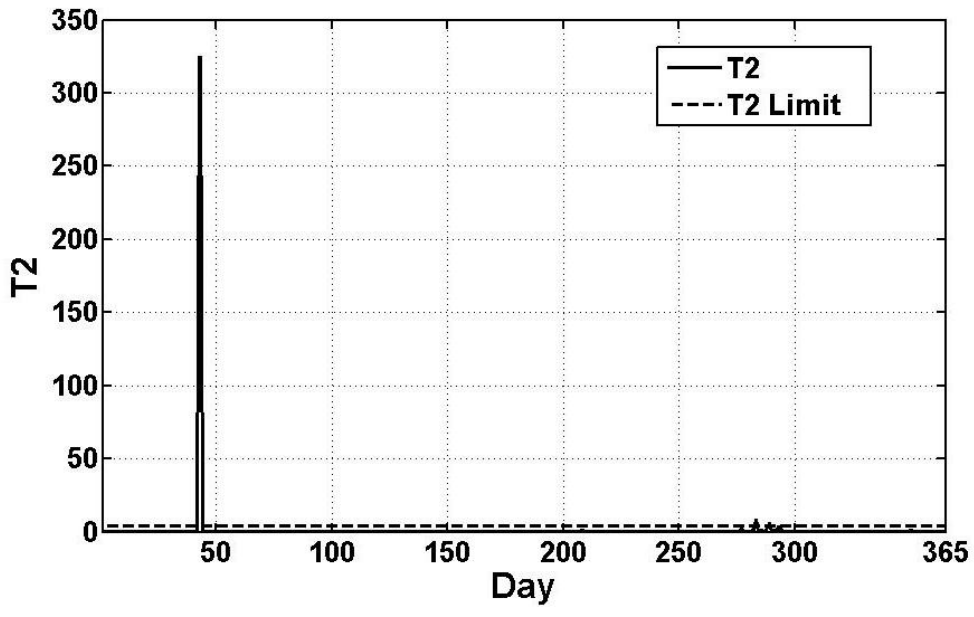

(b) Hotelling's $T^{2}$ index

FIGURE 7: Principal component analysis statistic measures for the back door entry sensor data (a) $S P E$ index, (b) Hotelling's $T^{2}$ index.

PCs based on the fuzzy outliers rank are shown in Figure 9-b and Figure 10-b. As shown in these figures, no extreme outliers are found in these data sets, there are only slight outliers (stars) and more or less change in her behaviour (circles). Therefore, an alarm should be sent to the carer only in the case of slight outliers to check if there is something wrong. More or less normal case can be considered as quite close to normal case. This case (MN) is used to distinguish between routine activities and slight change in behavioural activities.

Our experiments were repeated for data collected in case study II. Figure 11-a shows the scatter plots for the first and the second PCs of the binary data collected from the environment of the front door entry sensor of case study II. The corresponding labelled PCs based on the fuzzy outliers rank are shown in Figure 11-b. There is a significant difference between these two groups. The first group shows the normal data (circles) since their $S P E$ and Hotelling's $T^{2}$ are less than the confidence limits, while the second group shows the extreme outliers or anomalies (squares) as their $S P E$ and Hotelling's $T^{2}$ are greater than the confidence limits. 


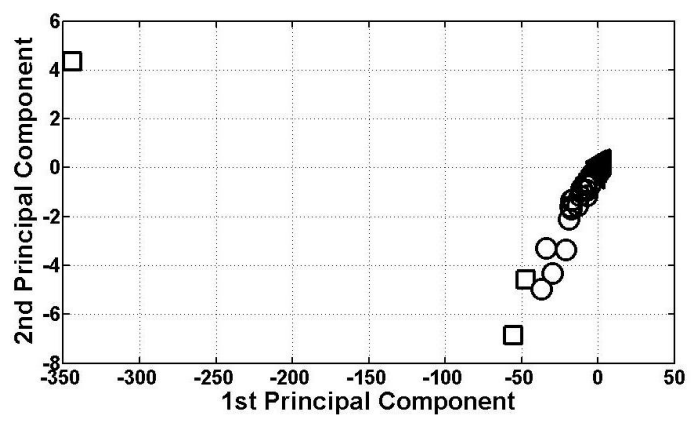

(a) Back door

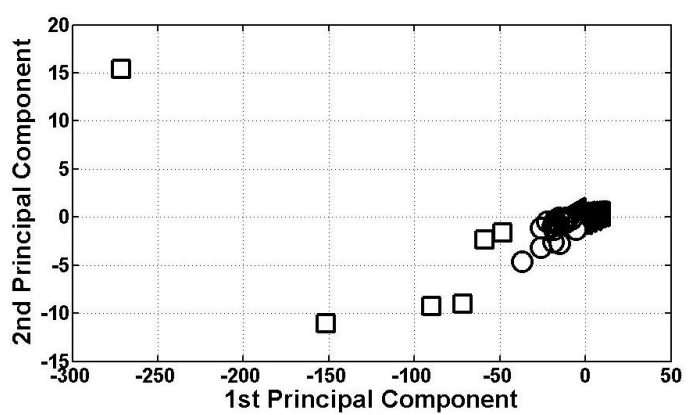

(b) Front door

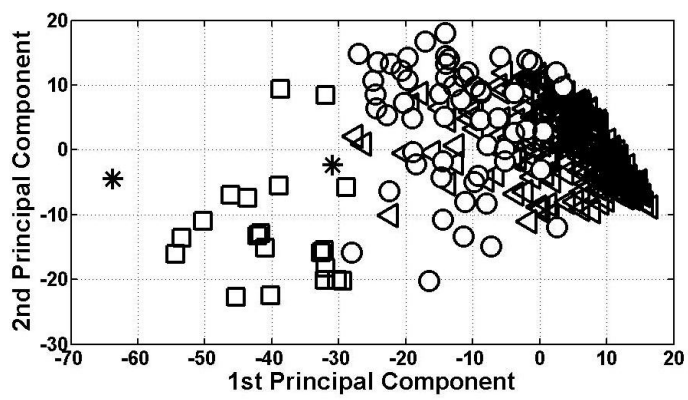

(c) Lounge

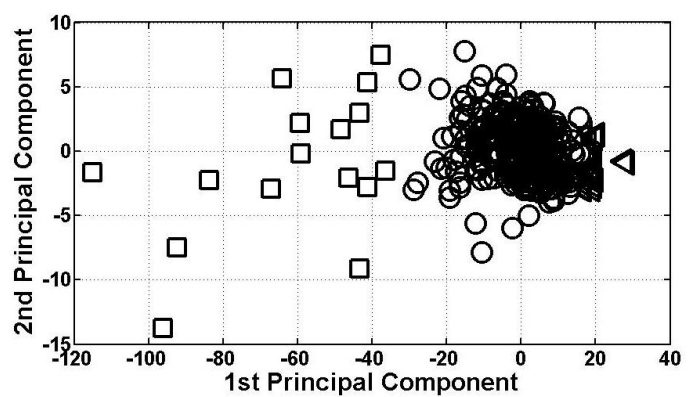

(d) Kitchen

FIGURE 8: Scattered plot for the 1st and 2nd principal components of the data used in case study I with classification. Triangles represent normal, squares represent extreme outliers, stars represent slight outliers and circles represent more or less normal pattern. 


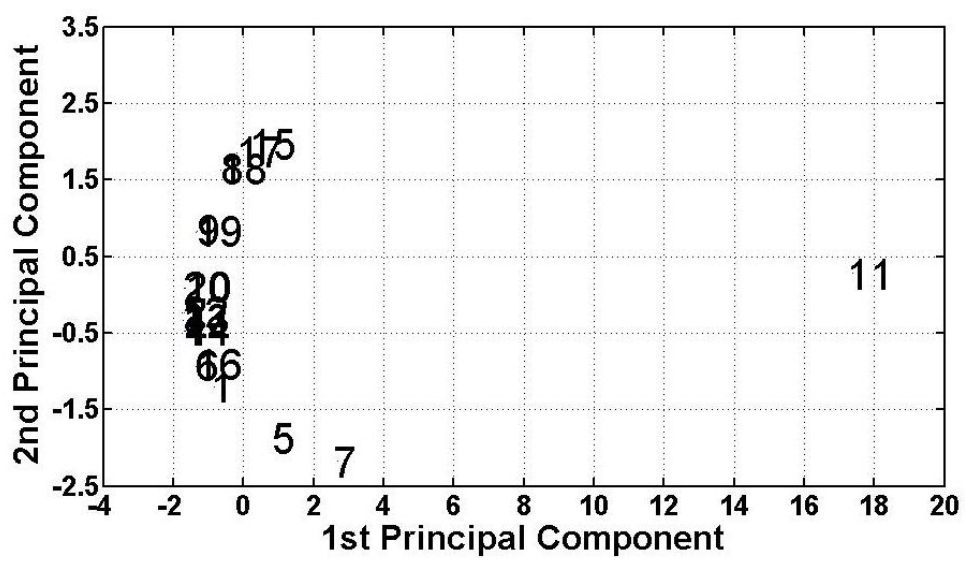

(a) Without classification

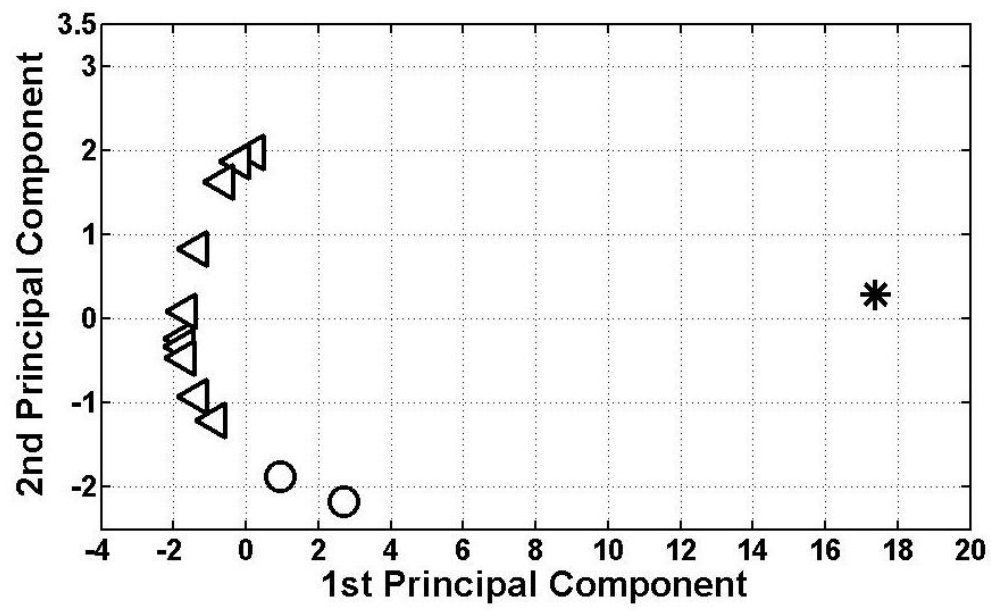

(b) With classification

FIGURE 9: Scattered plot for the 1st and 2nd principal components of the bedroom motion sensor data used in case study III. Triangles represent normal, squares represent extreme outliers, stars represent slight outliers and circles represent more or less normal pattern.

The results obtained from this real environment has shown that proposed outliers detection system can effectively distinguish between the normal and abnormal data.

\section{CONCLUSIONS AND FUTURE WORKS}

In this paper, investigation into the relationships between frequent patterns of user activities representing the activities of daily living is presented. To tackle the high dimensionality of the data and to identify the outliers and anomalies of user's activities, a two stages outlier detection system integrating PCA and FRBS is proposed. The principal components from the PCA analysis are used to identify two indices namely the residual SPE and Hotelling's $T^{2}$. Using these indices, the outliers and abnormal behaviour of an inhabitant are identified using FRBSs. The proposed system is proven to be able to classify the normal and abnormal behaviour or outliers. The severity of the outliers is also identified. The relationships between similar patterns are summarized in a meaningful manner by regularly generating a symbolic 


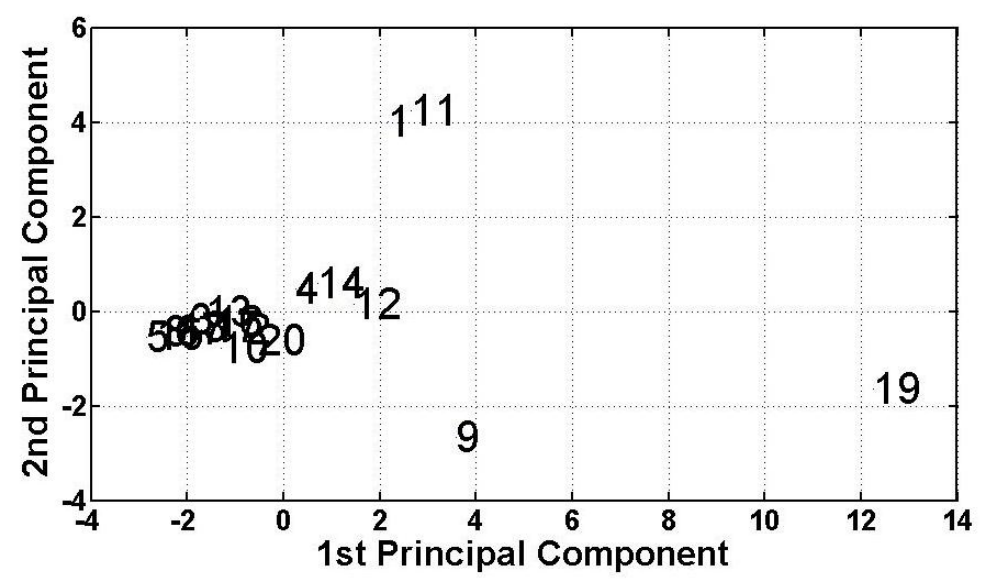

(a) Without classification

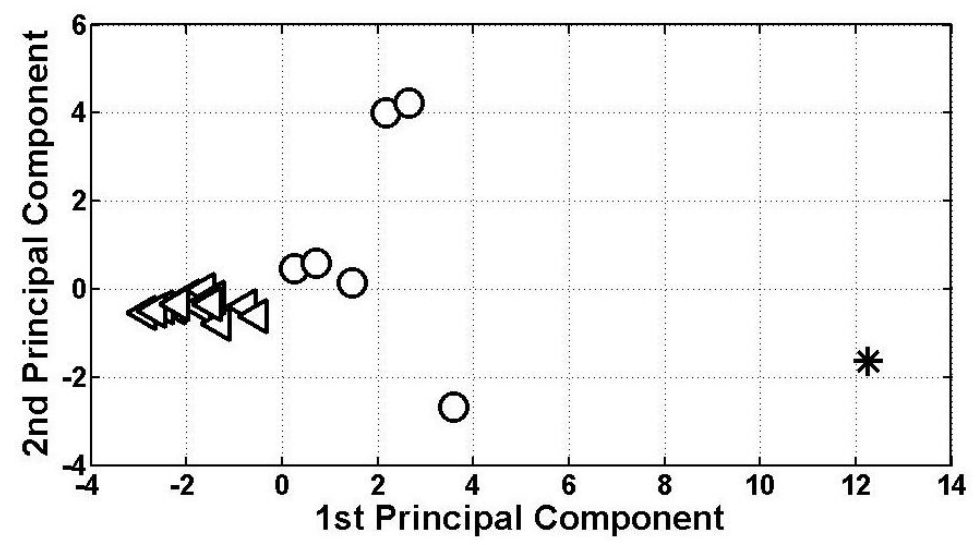

(b) With classification

FIGURE 10: Scattered plot for the 1st and 2nd principal components of the bathroom door data used in case study III.

report in natural language based on the extracted patterns. The proposed techniques are evaluated by applying to three real case studies.

For future work, the proposed technique is applied to better describe when and where the outliers and abnormalities occurred using the start time/end time of an activity and the location where the activity is happen. In this case, the Hamming distance computed using the duration of an activity within an hour rather than total hours in a day. The results and knowledge gained from this research are not limited to the field of intelligent environments only, and it could also be used in other domains such as wildlife monitoring and traffic monitoring.

\section{ACKNOWLEDGMENT}

Authors would like to thank Just Checking Ltd. (http://www.justchecking.co.uk) for their support of this work by providing the real data in the case studies. 


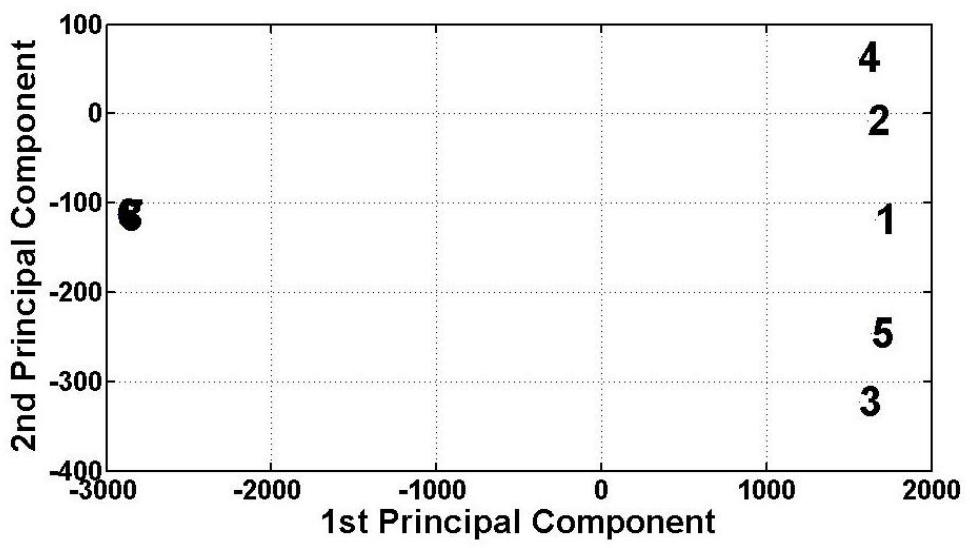

(a) Without classification

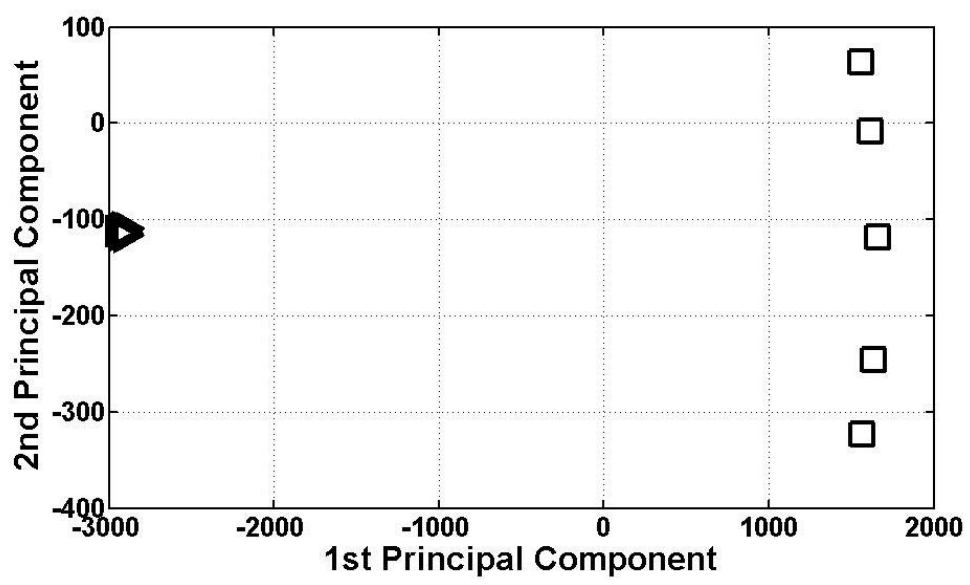

(b) With classification

FIGURE 11: Scattered plot for the 1st and 2nd principal components of the front door data used in case study II.

\section{REFERENCES}

AkHlaghinia, Mohammad JaVAD. 2010. Occupancy monitoring and prediction in ambient intelligent environment. Ph. D. thesis, Nottingham Trent University, Nottingham, UK.

Atallah, Louis, and GuAng-Zhong YANG. 2009. The use of pervasive sensing for behaviour profiling - a survey. Pervasive and Mobile Computing, 5(5):447-464.

Bouhouche, Salah, Malek Lahreche, Abdelkrim Moussaoui, and Jurgen Bast. 2007. Quality monitoring using principal component analysis and fuzzy logic application in continuous casting process. American Journal of Applied Sciences, 4:637-644.

Breunig, Markus M., HANS-Peter Kriegel, Raymond T. NG, and Jrg SANDER. 2000. Lof: Identifying density-based local outliers. Proceedings of ACM SIGMOD Int. Conf. on Mangement of Data, 27:1-12.

Cha, Sung Hyuk, Sungsoo Yoon, and C.C. TAPPERT. 2005. On binary similarity measures for handwritten character recognition. In Proceedings of the Eighth International Conference on Document Analysis and Recognition, Volume 1, pp. 4-8.

Chandola, Varun, Arindam Banerjee, and Vipin Kumar. 2009. Anomaly detection: A survey. ACM Computing Surveys (CSUR), 41.

Chen, Shiguo, and Daoqiang Zhang. 2011. Semisupervised dimensionality reduction with pairwise constraints for hyperspectral image classification. IEEE Geoscience and Remote Sensing Letters, 8(2):369- 
373.

Choi, Seung-Seok, Sung-Hyuk Cha, and Charles C. Tappert. 2010. A survey of binary similarity and distance measures. Journal of Systemics, Cybernetics and Informatics, 8(1):43-48.

CoOK, DiAne J. 2007. Making sense of sensor data. IEEE Pervasive Computing, 6(2):105-108.

Cook, Diane J., and Chiu-Che Tseng. 2006. Mining from time series human movement data. IEEE International Conference on Systems, Man and Cybernetics, 13:3241-3243.

Cunningham, PAdRaig. 2007. Dimension reduction. Technical Report UCD-CSI-2007-7, University College Dublin.

Das, Barnan, Chao Chen, Nairanjana Dasgupta, Diane Cook, and Adriyana M. Seelye. 2010. Automated prompting in a smart home environment. In IEEE International Conference on Data Mining Workshops, pp. 1045-1052.

Doctor, FaiYAz, HANi Hagras, and Victor CALlaghan. 2005. A fuzzy embedded agent-based approach for realizing ambient intelligence in intelligent inhabited environments. IEEE Transaction on Systems, Man, and Cybernetics-part A: Systems and Humans, 35:55-65.

Engel, DANiel, LARs Huttenberger, and Bernd Hamann. 2012. A survey of dimension reduction methods for high-dimensional data analysis and visualization. Proceedings of IRTG 1131 Workshop 2011, 27:135-149.

ERlich, Zippy, Roy Gelbard, and ISRAEL SPIEGLER. 2002. Data mining by means of binary representation:a model for similarity and clustering. Information Systems Frontiers, 4:187-197.

Han, Jiawei, and Micheline Kamber. 2006. Data Mining: Concepts and Techniques. Elsevier Inc. Publishing.

Huang, Anna. 2008. Similarity measures for text document clustering. In Proceedings of the Sixth New Zealand Computer Science Research Student Conference, pp. 49-56.

Illingworth, R., V. Callagha, and H. Hagras. 2006. Towards the detection of temporal behavioural patterns in intelligent environments. In 2nd IET International Conference on Intelligent Environments, pp. $119-125$.

Jain, Gaurav, Diane Cook, and Vikramaditya Jakkula. 2006. Monitoring health by detecting drifts and outliers for a smart environment inhabitant. In Proceedings of the 4th Int. Conf. on Smart Homes and Health Telematics.

JAKKUlA, Vikramaditya, and DiAne COOK. 2010. Outliers detection in smart environment structured power datasets. In Proceedings of the Sixth Int, Conf. on Intelligent Environments, pp. 29-33.

JAKKULA, ViKRAMADITYA, and DiANE COOK. 2011. Detecting anomalous sensor events in smart home data for enhancing the living experience. In Proceedings of the AAAI Workshop on Artificial Intelligence and Smarter Living: The Conquest of Complexity.

JustChecking. 2013. Supporting independence people with dementia, http://www . justchecking . Co.uk.

Kenner, A. 2008. Securing the elderly body: Dementia, surveillance, and the politics of aging in place. Surveillance and Society, 5:252-269.

Khan, Shehroz S., Michelle E. Karg, Jesse Hoey, and Dana Kuli. 2012. Towards the detection of unusual temporal events during activities using hmms. In Proceedings of the ACM Conference on Ubiquitous Computing, pp. 1075-1084.

Kumar, SAChin, VAsilis Sotiris, and Michael PeCht. 2008. Health assessment of electronic products using mahalanobis distance and projection pursuit analysis. International Journal of Computer and Information Engineering, 2:243-251.

LARA, OSCAR D., and MigUel A. LABRADOR. 2013. A survey on human activity recognition using wearable sensors. IEEE Communications Surveys and Tutorials, 15(3):1192-1209.

Li, Hui, Qingfan Zhang, and PeiYong DuAn. 2008. A novel one-pass neural network approach for activities recognition in intelligent environments. In Intelligent Control and Automation, 2008. WCICA 2008. 7th World Congress on, pp. 50-54.

LiU, Jun, Khiang-WeE Lim, Rajagopalan Srinivasan, and Xuan-Tien Doan. 2005. On-line process monitoring and fault isolation using pca. In Proceedings of the IEEE Int. Symposium on Intelligent Control, pp. 27-29.

Lotfi, Ahmad, Caroline Langensiepen, Sawsan M. Mahmoud, and M. J. Akhlaghinia. 2012. Smart homes for the elderly dementia sufferers: Identification and prediction of abnormal behaviour. Journal of Ambient Intelligence and Humanized Computing, 3(3):205-218. 
Mahmoud, Sawsan, Ahmad Lotfi, and Caroline Langensiepen. 2012. User activities outlier detection system using principal component analysis and fuzzy rule-based system. In Proc. of the Fifth International Conference on PErvasive Technologies Related to Assistive Environments (PETRA).

Manjunatha, P., A. K. Verma, and A. SRividya. 2008. Multi-sensor data fusion in cluster based wireless sensor networks using fuzzy logic method. In Proceeding of the 3rd International Conference on Industrial Information System, pp. 8-10.

Martin, Trevor, Basim Majeed, Beum-Seuk Lee, and Nick Clarke. 2006. Fuzzy ambient intelligence for next generation tele-care. In IEEE International Conference on Fuzzy Systems, pp. 16-21.

Medjahed, Hamid, Dan Istrate, Jerome Boudy, and Bernadete Dorizzi. 2009a. A fuzzy logic system for home elderly people monitoring. In Proc. of the 10th WSEAS International Conference on Fuzzy Systems (FS'09), pp. 69-75.

Medjahed, Hamid, Dan Istrate, Jerome Boudy, and Bernadette Dorizzi. 2009b. Human activities of daily living recognition using fuzzy logic for elderly home monitoring. In FUZZ IEEE Korea, pp. 20-24.

Mller, Wolfgang, Thomas Nocke, and Heidrun Schumann. 2006. Enhancing the visualization process with principal component analysis to support the exploration of trends. In Proceedings of the Conferences in Research and Practice in Information Technology, Volume 60.

Park, Sang-Ho, Ju-Hong Lee, Jae-Won Song, and TAe-Su Park. 2009. Forecasting change directions for financial time series using hidden markov model. Lecture Notes in Computer Science, Springer, 5589:184-191.

Rashidi, Parisa, Diane J. Cook, Lawrence B. Holder, and Maureen Schmitter-Edgecombe. 2011. Discovering activities to recognize and track in a smart environment. IEEE Transactions on Knowledge and Data Engineering, 23:527-539.

Rawat, Sanjay, V. P. Gulati, Arun K. Pujari, and V. Rao Vemuri. 2006. Intrusion detection using text processing techniques with a binary-weighted cosine metric. Journal of Information Assurance and Security, 1:43-50.

Singla, Geetika, Diane J. Cook, and Maureen Schmitter-Edgecombe. 2010. Recognizing independent and joint activities among multiple residents in smart environments. Journal of Ambient Intelligent Human Computing, 1:57-63.

Tapia, Emmanuel Munguia, Stephen S. Intille, and Kent Larson. 2004. Activity recognition in the home using simple and ubiquitous sensors. PERVASIVE, LNCS, Springer, Heidelberg, 3001:158-175.

van der MaAten, Laurens, Eric Postma, and JaAp van Den HeriK. 2009. Dimensionality reduction: A comparative review. Technical report, Tilburg University.

YAN, QIUYAN, SHIXIONG XIA, and YILONG SHI. 2010. An anomaly detection approach based on symbolic similarity. In Control and Decision Conference.

Ye, JiePing, and Shuiwang Ji. 2009. Discriminant Analysis for Dimensionality Reduction: An Overview of Recent Developments, Chapter 1. John Wiley and Sons Inc Hoboken NJ USA.

Ye, LI, ZHI-GUANG QIN, JUAN WANG, and JING Jin. 2010. Anomaly event detection in temporal sensor network data of intelligent environments. In Proc. of the 2nd Int. Conf. on Computer Engineering and Technology, Volume 7, pp. 414-420.

Yogurtcu, Osman, Engin Erzin, and Attila Gursoy. 2006. Extracting gene regulation information from micro array time-series data using hidden markov models. Lecture Notes in Computer Science, Springer, 4263:144-153.

Zheng, Huiru, Member, HaiYing WANG, and Norman Black. 2008. Human activity detection in smart home environment with self-adaptive neural networks. In IEEE International Conference on Networking, Sensing and Control, pp. 1505-1510. 